\title{
Interference Estimation for Admission Control in Multi-service DS-CDMA Cellular Systems
}

\author{
Lei Zhuge and Victor O. K. Li \\ Department of Electrical and Electronic Engineering \\ The University of Hong Kong \\ Pokfulam Road, Hong Kong \\ \{lzhuge,vli\}@eee.hku.hk
}

\begin{abstract}
Wideband code division multiples access (CDMA) is one of the major options for the next generation mobile cellular system. However, there is only limited research on the admission control for CDMA systems containing multiple service classes. In this paper the multi-service CDMA admission control problem is addressed by an approach which is conceptually simple, and yet produces satisfactory results. In our approach the limit on the acceptable interference level in a cell is translated into a constraint on the number of users of each service class in the local and neighboring cells. The randomness of user locations, shadowing and imperfect power control is captured as a whole by a log-normal distribution. Simulation results show that this approach is quite accurate over a wide range of required system outage probabilities.
\end{abstract}

\section{INTRODUCTION}

The direct sequence code division multiple access (DSCDMA) cellular system IS-95 was developed and successfully introduced into the market in the 1980s and 1990s. Since then DS-CDMA technology has grown rapidly. As two wideband CDMA proposals (WCDMA [6] and cdma2000 [7]) have been approved as the International Mobile Telecommunications (IMT-2000 [5]) specifications, DS-CDMA systems are expected to play a major role in the next generation mobile communication systems.

While much effort has been placed on the physical layer research and development, there is relatively limited research on system level issues such as capacity analysis, call admission (access) control, burst admission, and quality of service for multiservice CDMA systems [8]-[16]. Among these issues admission control has received the most attention.

The majority of existing work on multi-service CDMA admission control is on the integration of data and voice services [8]-[11]. The focus is on the tradeoff between the number of voice and data users according to their blocking or outage probability requirements. As the aim of wideband CDMA is to support various services with a wide range of data rates (e. g. $2.4 \mathrm{kbps}-2 \mathrm{Mbps}$ ), we need a more general analysis on the multi-service system containing an arbitrary number of service classes.

The interference limit in a CDMA cell can be transformed into a bound on the number of users, as shown by Gilhousen et al. [1] and Viterbi [2]. When considering shadowing and imperfect power control effects, this bound becomes soft and the "softness" is dependent on the outage probability in the system. In this paper we extend these results to a constraint on the number of users in all service classes in the general multi-service

This research is supported in part by the Research Grants Council, Hong Kong, under Grant No. HKU 7225/99E.
CDMA system. The resultant constraint, based on a new lognormal approximation instead of the conventional Gaussian approximation or Chemoff bound, is not only simple to calculate, but also quite accurate.

The general constraint is derived in Section III. Before that we discuss a simpler case with perfect power control and no shadowing in Section II, which forms a basis for the general constraint formulation. Our results are validated by the numerical results in Section IV. Section V gives conclusions and directions for future work.

\section{A STATic MOdEL}

In this section we temporarily disregard the randomness of user signal powers caused by shadowing and imperfect power control in a cellular CDMA system, and derive a constraint on the number of users in each service class based on interference estimation. The result will be used in the next section where the static assumption is relaxed.

The parameter used to determine the acceptable interference level in a CDMA cell is the bit-energy to interference-density ratio $\gamma$, calculated as

$$
\gamma_{k} \triangleq \frac{\left(E_{b}\right)_{k}}{I_{0}}=\frac{S_{k}}{I} \frac{W}{R_{k}} \quad(k=1, \ldots, L)
$$

where the subscript $k$ denotes parameters for the $k$-th service class, $L$ is the number of service classes in the cell, $S_{k}$ is the received user signal power at the base station, $I$ is the total interference power received at the base station, $W$ is the system bandwidth, $R_{k}$ is the data rate of the application, $\left(E_{b}\right)_{k}=S_{k} / R$ is the bit energy in the received signal, and $I_{0}=I / W$ is the received interference density.

In order to guarantee good communication quality for the $k$-th service class, $\gamma_{k}$ must be greater than a threshold value $\gamma_{k}^{*}$. For example, in the current IS-95 system with convolutional coding, interleaving, and multipath combining in the RAKE receiver, a $\gamma_{k}^{*}$ of $6 \mathrm{~dB}$ can guarantee a bit error rate (BER) of $10^{-3}$.

Assume perfect power control, i. e. $\gamma_{k}=\gamma_{k}^{*}$, then the received signal power $S_{k}$ is the same for all users of service class $k$. The total interference (plus noise) power received at the base station is hence approximately formulated as ${ }^{1}$

$$
I=\sum_{k=1}^{L} N_{k} S_{k}+S_{\text {out }}+n_{0} W
$$

${ }^{1}$ This approximation is valid when the signal power from any signal user is small compared with the total interference power. 


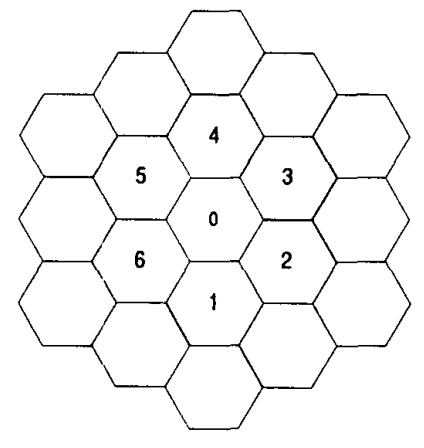

Fig. 1. Cell layout for interference analysis

where $N_{k}$ is the number of users from the $k$-th service class in the local cell, $S_{\text {out }}$ is the total interference power received from neighboring cells, and $n_{0}$ is the power density of thermal noise.

(1) also means $S_{k} /\left(R_{k} \gamma_{k}\right)=I / W$, hence from (2) ${ }^{2}$

$$
\begin{aligned}
I & =\sum_{k=1}^{L} N_{k} R_{k} \gamma_{k}\left(\frac{S_{k}}{R_{k} \gamma_{k}}\right)+S_{\text {out }}+n_{0} W \\
& =\left(\sum_{k=1}^{L} N_{k} R_{k} \gamma_{k}\right) \frac{I}{W}+S_{\text {out }}+n_{0} W
\end{aligned}
$$

and we get

$$
\sum_{k=1}^{L} N_{k} R_{k} \gamma_{k}=W\left(1-\frac{S_{\text {out }}+n_{0} W}{I}\right)
$$

Due to the dynamic range limitation of the receiver, there exists an upper bound on the total received interference power, expressed as the noise-density to interference-density ratio [2]

$$
\frac{n_{0}}{I_{0}}>\eta \text { or } \frac{n_{0} W}{I}>\eta
$$

where $\eta<1$ is dependent on the system design ( $\eta$ is typically chosen between 0.1 and 0.25 in the IS-95 system [2]).

Adding this constraint, (3) becomes

$$
\sum_{k=1}^{L} N_{k} R_{k} \gamma_{k}<W\left(1-\eta-\frac{S_{\text {out }}}{I}\right)
$$

Next we relate $S_{\text {out }} / I$ to the number of users in the neighboring cells. For simplicity here we only consider the first tier of six neighboring cells (cells 1 to 6 in Fig. 1), though the method presented here can be generally applied to any number of tiers. Assume perfect power control in each cell. $S_{\text {out }}$ is then the total signal power from all users of all service classes in the six neighboring cells received by the base station in the local cell (cell 0), i. e.

$$
S_{\text {out }}=\sum_{c=1}^{6} \sum_{k=1}^{L_{c}} \sum_{i=1}^{N_{k c}} \alpha_{k i c} S_{k c}
$$

${ }^{2}$ For convenience, here we use $\gamma_{k}$ for $\gamma_{k}^{*}$. where $S_{k c}$ is the power of class- $k$ user received by the base station of cell $c, N_{k c}$ is the number of class- $k$ users in cell $c, L_{c}$ is the number of service classes in cell $c$, and $\alpha_{k i c}$ is the path-loss ratio for user $i$ of class $k$ in cell $c$ (the ratio of the path loss from the user to the base station of cell $c$ to that from the user to the base station of cell 0 ). Determination of the path loss ratio $\alpha_{k i c}$ will be discussed in the next section.

From (1) we know that $S_{k} / I=\left(R_{k} \gamma_{k}\right) / W$ is independent of signal powers. In order to apply this to $S_{\text {out }} / I$ in (5), (note that the $I$ here is the interference power received by the base station in cell 0 ) we assume that the interference power $I$ is approximately the same in each cell. This assumption is valid here because we are trying to find the boundary constraints on the number of users when the system capacity is reached. Since all cells are assumed to have the same area and bandwidth allocation, the total acceptable interference level should be approximately the same in each cell if power control is performed over the same service class. This assumption is also supported by the dynamic power range limitation at the receiver (4). Please note, however, that the homogeneous interference assumed here does not necessarily mean homogeneous traffic load in each cell. It can happen in heterogeneous traffic conditions in which a cell has less traffic load than another one but receives more interference from neighboring cells, and the total interference level in the two cells is about the same.

With the same $I$ in each cell, we get from (6)

$$
\begin{aligned}
\frac{S_{\text {out }}}{I} & =\sum_{c=1}^{6} \sum_{k=1}^{L_{c}} \sum_{i=1}^{N_{k c}} \alpha_{k i c} \frac{S_{k c}}{I} \\
& =\frac{1}{W} \sum_{c=1}^{6} \sum_{k=1}^{L_{c}} \sum_{i=1}^{N_{k c}} \alpha_{k i c} R_{k c} \gamma_{k c}
\end{aligned}
$$

Applying (7) in (5), we obtain the constraint on the number of users under this static model of perfect power control and no shadowing

$$
\sum_{k=1}^{L_{0}} N_{k 0} R_{k 0} \gamma_{k 0}+\sum_{c=1}^{\theta} \sum_{k=1}^{L_{c}} \sum_{i=1}^{N_{k c}} \alpha_{k i c} R_{k c} \gamma_{k c}<W(1-\eta)
$$

where we have added a second subscript 0 to each parameter of the first term in (5) to denote cell 0 . Although we can find the average value of the path loss ratio for all other-cell users, we prefer leaving it to later discussion where its randomness is included in the statistical version of (8).

(8) is a simple linear constraint (with the path loss ratio $\alpha_{k i c}$ unsolved yet). From (8) we see that $R_{k} \gamma_{k}$ can be regarded as the fraction of system bandwidth $W$ "effectively utilized" by a user of service class $k$. (The consumption of $W$ by an outside user is reduced by the path loss ratio.) Hence the left side of (8) is an index of system bandwidth utilized, defined by a parameter C

$$
C \triangleq \sum_{k=1}^{L_{0}} N_{k 0} R_{k 0} \gamma_{k 0}+\sum_{c=1}^{6} \sum_{k=1}^{L_{e}} \sum_{i=1}^{N_{k c}} \alpha_{k i c} R_{k c} \gamma_{k c}
$$

In addition, since $R_{k} \gamma_{k}$ is proportional to $S_{k} / I, C$ is also a good estimate of the relative interference level in the cell. In fact, by dividing both sides of (8) by $W$, we can easily restore the interference expression (2). 


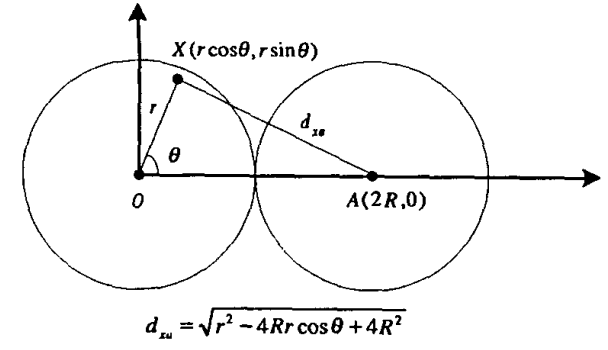

Fig. 2. Calculation of path loss ratio

\section{REAL SYSTEM CONSIDERATIONS}

In real CDMA systems user signal powers received at the base station is generally random due to variable user locations, the shadowing effect, and imperfect power control. Hence the static constraint derived in the last section must be modified to accommodate these random factors.

\section{A. Key Random Factors}

Path loss ratio - The first random factor to be considered is the path loss ratio $\alpha$ introduced in the last section. Since the path loss ratio is related to the user location which is usually unpredictable, the exact value of the path loss ratio can not be determined for each user. Here we use the approximation method provided in [12], [17] to analyze the statistical behavior of the path loss ratio. The accuracy of this approximation is verified by simulations in [12], [17].

Assume user locations are uniformly distributed in circular cells. Since the path loss of signal power is proportional to the $m$-th power of the distance the signal travels (typically $m=4$ in the cellular environment), the path loss ratio for a user at location $X$ in Fig. 2 is equal to $\left(r / d_{x a}\right)^{m}$ where $d_{x a}$ is the distance between the user and the target base station at $A$. (Here the user is power controlled by the base station at $O$.) The mean and variance of the path loss ratio are readily calculated as (taking $m=4$ )

$$
\begin{aligned}
\mathrm{E}[\alpha] & =\int_{\theta=0}^{2 \pi} \int_{r=0}^{R}\left(\frac{r}{\sqrt{r^{2}-4 R r \cos \theta+4 R^{2}}}\right)^{m} \\
& \left(\frac{r}{\pi R^{2}}\right) d r d \theta \\
& 0.0474 \\
\operatorname{Var}[\alpha]= & \int_{\theta=0}^{2 \pi} \int_{r=0}^{R}\left(\frac{r}{\sqrt{r^{2}-4 R r \cos \theta+4 R^{2}}}\right)^{2 m} \\
& \left(\frac{r}{\pi R^{2}}\right) d r d \theta-\mathrm{E}^{2}[\alpha] \\
= & 0.0121
\end{aligned}
$$

Shadowing - Shadowing is caused by the reflection and diffraction of the transmitted signal due to different terrain conditions and large objects. The effect of shadowing is a variation of the path loss in addition to the $m$-th power rule. The shadowing factor on the path loss is usually modeled as a log-normal random variable $\epsilon$ with parameters $\left(0, \sigma_{\epsilon}^{2}\right)$, or equivalently, the decibel value of this factor $10 \log _{10} \epsilon$ has a Gaussian distribution with mean 0 and variance $\sigma_{\epsilon}^{2}$, where $\sigma_{\epsilon}=8 \mathrm{~dB}$ is a typical value [2], [3].

It has been shown that the shadowing factor $\epsilon$ is independent of the path loss ratio $\alpha$. The shadowing factor of each user is also assumed independent of each other since user locations are assumed independent of each other.

Imperfect power control - Imperfect power control is caused by errors in the power control loop. Under imperfect power control the bit-energy to interference-density ratio $\gamma$ determined by (1) has a random value varying around the target $\gamma^{*}$. The deviation of $\gamma$ from $\gamma^{*}$ is usually modeled as a log-normal random variable $\pi[2],[17]$, i. e. $^{3}$

$$
\gamma=\pi \gamma^{*} \quad \text { where } \quad \pi \sim \operatorname{Ln}\left(0, \sigma_{\pi}^{2}\right)
$$

Typically $\sigma_{\pi}$ is in the range of $1.5-2.5 \mathrm{~dB}$. The imperfect power control factor $\pi$ of each user is independent of each other.

\section{B. The General Constraint}

Considering the random factors described above, the system bandwidth utilization defined in (9) should be modified to its statistical version as

$$
C=\sum_{k=1}^{L_{0}} \sum_{i=1}^{N_{k 0}} \pi_{k i 0} R_{k 0} \gamma_{k 0}^{*}+\sum_{c=1}^{6} \sum_{k=1}^{L_{c}} \sum_{i=1}^{N_{k c}} \alpha_{k i c} \epsilon_{k i c} \pi_{k i c} R_{k c} \gamma_{k c}^{*}
$$

where $\alpha_{k i c}, \epsilon_{k i c}$, and $\pi_{k i c}$ are path loss ratio, shadowing factor and imperfect power control factor for user $i$ of class $k$ in cell $c$, respectively. $\left\{\alpha_{k i c}\right\}$ are independent, identically distributed (i. i. d.) random variables each having distribution as described in (10); $\left\{\epsilon_{k i c}\right\}$ are i. i. d. random variables each having distribution $\operatorname{Ln}\left(0, \sigma_{\epsilon}^{2}\right)$; and $\left\{\pi_{k i c}\right\}$ are i. i. d. random variables each having distribution $\operatorname{Ln}\left(0, \sigma_{\pi}^{2}\right)$.

The static constraint (8) should also be modified to a constraint on the system outage probability, or the probability of the total interference in the system exceeding the acceptable level. From the discussion in Section II we know that this outage probability is just the probability of the event $[C \geq W]$, hence it is required that

$$
\mathrm{P}[C \geq W]<\delta
$$

where $\delta$ is dependent on system design. Here for convenience we assume that the dynamic power range limitation (4) has been absorbed in $W$, i. e. the system bandwidth has already been reduced by the factor $(1-\eta)$.

In order to find the constraint on the number of users from (12) and (13), we need to analyze the tail probability of $C$ based on the statistical properties of random factors $\alpha, \epsilon$, and $\pi$.

It is easily proved that the product of two $\log$-normal random variables $\epsilon \sim \operatorname{Ln}\left(0, \sigma_{\epsilon}^{2}\right)$ and $\pi \sim \operatorname{Ln}\left(0, \sigma_{\pi}^{2}\right)$ is another lognormal random variable $\zeta \sim \operatorname{Ln}\left(0, \sigma_{\zeta}^{2}\right)$ where $\sigma_{\zeta}^{2}=\sigma_{\epsilon}^{2}+\sigma_{\pi}^{2}$.

The mean and variance of $\epsilon_{\pi}$, where $\zeta \triangleq \epsilon \pi$, are

$$
\mathrm{E}[\zeta]=e^{\beta^{2} \sigma_{\zeta}^{2} / 2}, \operatorname{Var}[\zeta]=e^{2 \beta^{2} \sigma_{\zeta}^{2}}-e^{\beta^{2} \sigma_{\zeta}^{2}}
$$

${ }^{3} \operatorname{Ln}\left(0, \sigma_{\pi}^{2}\right)$ stands for $\log$-normal distribution with parameters $\left(0, \sigma_{\pi}^{2}\right)$, where $\sigma_{\pi}^{2}$ is the variance of the decibel value of the random variable. 
where $\beta=\ln 10 / 10$ is a constant.

The product of $\alpha$ and $\zeta$ is defined here as a random variable $\xi$. We do not know the distribution of $\xi$, but we only need its mean and variance

$$
\begin{gathered}
\xi \triangleq \alpha \zeta=\alpha \epsilon \pi \\
\mathrm{E}[\xi]=\mathrm{E}[\alpha] \mathrm{E}[\zeta], \quad \operatorname{Var}[\xi]=\mathrm{E}\left[\alpha^{2}\right] \mathrm{E}\left[\zeta^{2}\right]-\mathrm{E}^{2}[\alpha] \mathrm{E}^{2}[\zeta]
\end{gathered}
$$

where $E[\zeta]$ and $E\left[\zeta^{2}\right]$ can be obtained from (14), and $E[\alpha]$ and $\mathrm{E}\left[\alpha^{2}\right]$ can be obtained from (10).

Our problem (13) is the control of the tail probability of $C$ which is the sum of many random variables. Usually this kind of problems is solved by using Gaussian approximation or Chernoff bound [2], [8], [12], [13]. It is shown in [2], [12] that Chernoff bound is too conservative for the sum of log-normal random variables as it overestimates the tail probability by "an order of magnitude." While the Gaussian approximation produces good results in [2], we have shown by simulations that it is not accurate enough in our case where there exist sum and product of different kinds of random factors.

By many simulations (some of which are shown in the next section) we find that the tail probability of $C$ seems dominated by the log-normal component with the largest parameter $\sigma$ in $C$. Here the log-normal component with the largest $\sigma$ is $\zeta=\epsilon \pi$. More specifically, in terms of tail probability,

$$
C \approx A \zeta+B
$$

where $\zeta \sim \operatorname{Ln}\left(0, \sigma_{\zeta}^{2}\right)$ as in (14), $A$ and $B$ are properly chosen so that $(A \zeta+B)$ has the same mean and variance as $C$.

While we are still searching for analytical proof of this observation, the result looks pretty reasonable because a log-normal distribution has longer tail than a Gaussian distribution with the same mean and variance, and the tail becomes longer as its parameter $\sigma$ increases.

The mean and variance of $C$ are calculated as

$\mathrm{E}[C]=\mu_{0} \mathrm{E}[\pi]+\mu_{1} \mathrm{E}[\xi], \operatorname{Var}[C]=\sigma_{0}^{2} \operatorname{Var}[\pi]+\sigma_{1}^{2} \operatorname{Var}[\xi]$

where

$$
\begin{array}{ll}
\mu_{0}=\sum_{k=1}^{L_{0}} N_{k 0} R_{k 0} \gamma_{k 0}^{*}, & \sigma_{0}^{2}=\sum_{k=1}^{L_{0}} N_{k 0}\left(R_{k 0} \gamma_{k 0}^{*}\right)^{2} \\
\mu_{1}=\sum_{c=1}^{6} \sum_{k=1}^{L_{c}} N_{k c} R_{k c} \gamma_{k c}^{*}, & \sigma_{1}^{2}=\sum_{c=1}^{6} \sum_{k=1}^{L_{c}} N_{k c}\left(R_{k c} \gamma_{k c}^{*}\right)^{2}
\end{array}
$$

$\mathrm{E}[\xi]$ and $\operatorname{Var}[\xi]$ are given in (15), while $\mathrm{E}[\pi]$ and $\operatorname{Var}[\pi]$ can be obtained similarly as in (14).

Therefore, $A$ and $B$ in (16) are chosen as

$$
\begin{aligned}
& A=\sqrt{\frac{\sigma_{0}^{2} \operatorname{Var}[\pi]+\sigma_{1}^{2} \operatorname{Var}[\xi]}{\operatorname{Var}[\zeta]}} \\
& B=\mu_{0} \mathrm{E}[\pi]+\mu_{1} \mathrm{E}[\xi]-A \mathrm{E}[\zeta]
\end{aligned}
$$

By using the log-normal approximation (16), we can obtain the general constraint on the number of users $\left\{N_{k c}\right\}$ from the tail probability bound (13). If $\tau$ defines tail probability $\delta$ for $\zeta \sim \operatorname{Ln}\left(0, \sigma_{\zeta}^{2}\right)$, i. e. ${ }^{4}$

$$
\int_{\tau}^{\infty} \frac{1}{x \beta \sqrt{2 \pi} \sigma_{\zeta}} \exp \left[-\frac{\left(10 \log _{10} x\right)^{2}}{2 \sigma_{\zeta}^{2}}\right]=\delta
$$

the outage probability requirement (13) is achieved when

$$
A \tau+B<W
$$

or

$$
\mu_{0} \mathrm{E}[\pi]+\mu_{1} \mathrm{E}[\xi]+(\tau-\mathrm{E}[\zeta]) \sqrt{\frac{\sigma_{0}^{2} \operatorname{Var}[\pi]+\sigma_{1}^{2} \operatorname{Var}[\xi]}{\operatorname{Var}[\zeta]}}<W
$$

Since the means and variances of the random factors $\pi, \xi$, and $\zeta$ are all constants, and $\tau$ is constant, (20) defines a quadratic constraint on $\left\{N_{k c}\right\}$ (included in $\mu_{0}, \mu_{1}, \sigma_{0}$, and $\sigma_{1}$ ). The accuracy of this constraint is justified by simulations described in the next section.

This derivation of the general constraint can be extended to any system layout including more tiers of interfering cells, by adding corresponding terms to (20). Please note, however, that (20) requires exchange of knowledge $\left\{N_{k c}\right\}$ between neighboring cells, and evaluation of admission condition in all neighboring cells being considered as well as the local cell; hence inclusion of more tiers means more overhead in transmission and processing of admission data. Since the cells outside the first tier contributes only about $3 \%$ of the total interference in the local cell (for homogenous traffic load [17]), we think (20) gives enough information on interference estimation for admission control.

(20) is a major constraint which should be followed in admission control because it is directly drawn from the interference limit in the cell. Other constraints, such as the minimum required number of users of a certain service class, fairness among users, priorities of service classes, etc., may also be added to meet the needs of real applications.

\section{NUMERICAL RESULTS}

Consider a system with homogeneous traffic load. Each cell contains three service classes, and the number of users in a certain service class is the same in all cells. The system and traffic parameters used in the simulation are listed in Table 1 . The activity factors of the service classes are not considered here, although they can be easily included.

By fixing the bandwidth limit as $9 \mathrm{MHz}$ (10\% discount for the noise-density to interference-density ratio limit $\eta$ ), we now consider the change of the outage probability with respect to the number of users in different service classes (denoted as $N_{i}$ for service class $i$ ). Fig. 3 shows the outage probabilities obtained from Log-normal approximation, Gaussian approximation, and simulation. Simulation is done by generating random numbers (100,000 samples for each of the random factors $\alpha, \epsilon$. and $\pi$ in (12)) and measuring the proportion of those samples of $C$ exceeding $W$ as in (13). The computation time for each simulation

${ }^{4}$ There exist different expressions for $\log$-normal distribution. Here we use the version defined in [2] and [3]. 
TABLE I

SIMULATION PARAMETERS

\begin{tabular}{|c|c|c|}
\hline item & symbol & value \\
\hline system bandwidth & $W$ & $10 \mathrm{MHz}$ \\
$n_{0} / I_{0}$ ratio & $\eta$ & 0.1 \\
shadowing factor & $\sigma_{\epsilon}$ & $8 \mathrm{~dB}$ \\
imperfect PC factor & $\sigma_{\pi}$ & $2.5 \mathrm{~dB}$ \\
path loss ratio & $(\mathrm{E}[\alpha], \operatorname{Var}[\alpha])$ & $(0.0474,0.0121)$ \\
service class 1 & $\left(R_{1}, \gamma_{1}\right)$ & $(5 \mathrm{kbps}, 6 \mathrm{~dB})$ \\
service class 2 & $\left(R_{2}, \gamma_{2}\right)$ & $(10 \mathrm{kbps}, 6 \mathrm{~dB})$ \\
service class 3 & $\left(R_{3}, \gamma_{3}\right)$ & $(100 \mathrm{kbps}, 6 \mathrm{~dB})$ \\
\hline
\end{tabular}

point is three to four minutes using MATLAB on a shared Sun E4500 server. We can see that the outage probability is more sensitive to the number of high speed $(100 \mathrm{kbps})$ users than to the number of low speed (10 kbps) users. For the given $N_{1}$ and $N_{2}$, no more than seven high speed users $\left(N_{3}\right)$ can be admitted into the system if we want to keep the outage probability below 0.1 . However, the increase of outage probability with the number of $10 \mathrm{kbps}$ users $\left(N_{2}\right)$ is much slower. This observation suggests that we may use different admission policies for high speed and low speed users. For high speed users a hard limit is preferred because they take up much system resources. For low speed users, on the other hand, we can specify a soft capacity so that such users can take advantage of the varying interference level in the system.

Next we look at some examples with heterogeneous traffic load among the cells. In Fig. 4(a) the traffic load in the local cell is fixed, and the number of high speed users in each neighboring cell $\left(N_{3}^{\prime}\right)$ changes simultaneously (assuming these cells can always accommodate the increased traffic). On the contrary, in Fig. 4(b) we keep the traffic load in the neighboring cells unchanged, and increase the number of high speed users $\left(N_{3}\right)$ in the local cell. Other parameters are exactly the same in both cases. As a result, the two figures display similar range of $N_{3}$ and $N_{3}^{\prime}$ for small outage probabilities (below 0.1 ), with a little more high speed users admitted in the fixed neighbor-traffic case. In other words, the total interference of high speed users from all neighboring cells is a little stronger than that of high speed users in the local cell, under our simulation conditions.

\section{CONCLUSIONS AND FUTURE WORK}

In this paper we have presented a method to directly relate the interference level or bandwidth utilization in a multi-service DS-CDMA cell to the number of users from each service class in the local and neighboring cells. The aggregate effects of random user locations, shadowing and imperfect power control on our estimation are approximated as log-normal distributed. Then a constraint on the number of users is derived with respect to the outage probability. The resultant constraint has low computational complexity and satisfactory accuracy as shown by simulation results. Hence we think it is very effective in admission control for multi-service CDMA systems.

Some other issues will be addressed in our future work to refine the admission constraint. The first issue is soft handoff. When a mobile user is in the cell boundary region, it is

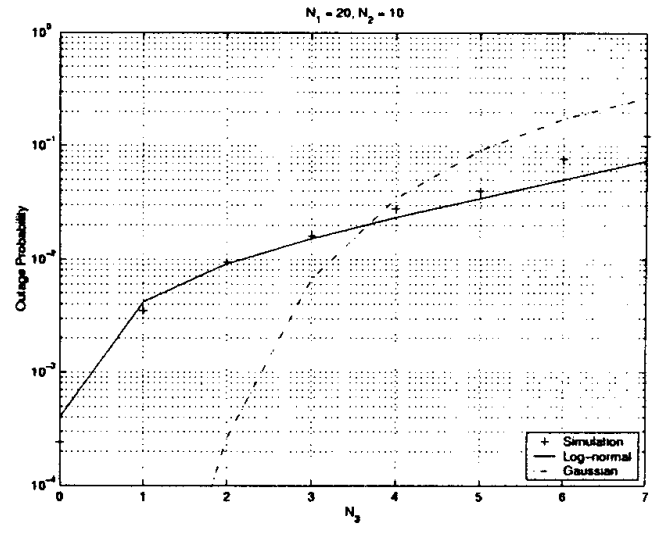

(a) fixed $N_{1}$ and $N_{2}$

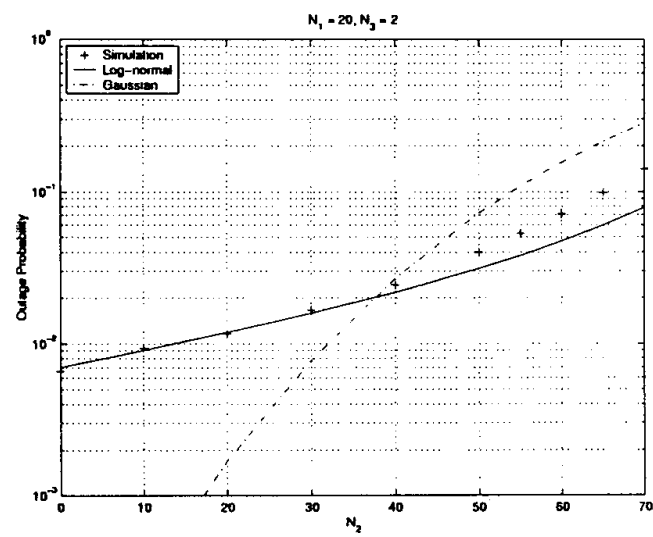

(b) fixed $N_{1}$ and $N_{3}$

Fig. 3. Outage probability versus number of users

power controlled by the base station receiving its signal with stronger power. This base station is not necessarily the nearest one. The shadowing factor is hence reduced, and its effect on the tail probability of the overall bandwidth utilization will be studied.

The other issue is variable user data rates. While it is beiter to control the number of high speed users under a hard limit, as mentioned in the last section, those low speed users may enjoy the soft capacity of DS-CDMA. Determination of the soft capacity is related to the user traffic models [2], [12], and hence it will be desirable to integrate the traffic models (e. g. on-off model, Markov-modulated Poisson process model, etc.) into our lognormal framework.

\section{REFERENCES}

[1] K. S. Gilhousen, I. M. Jacobs, R. Padovani, A. J. Viterbi, L. A. Weaver, and C. E. Wheatley, "On the capacity of a cellular CDMA system," IEEE Trans. Vehicul. Tech., vol. 40, no. 2, May 1991, pp. 303-312. 


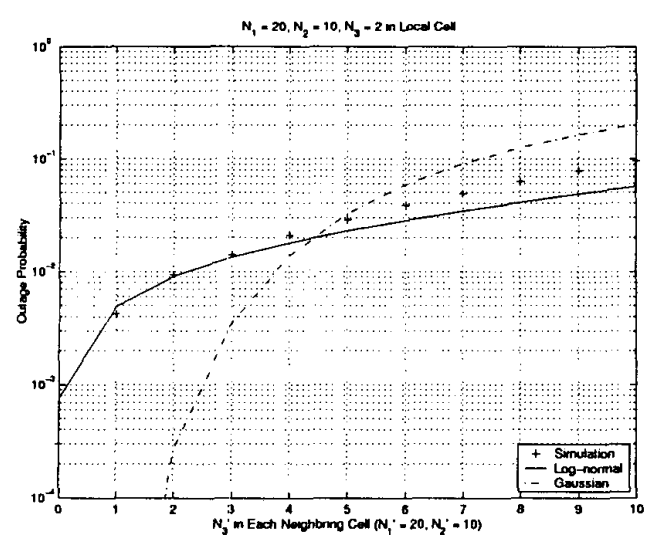

(a) fixed traffic in local cell

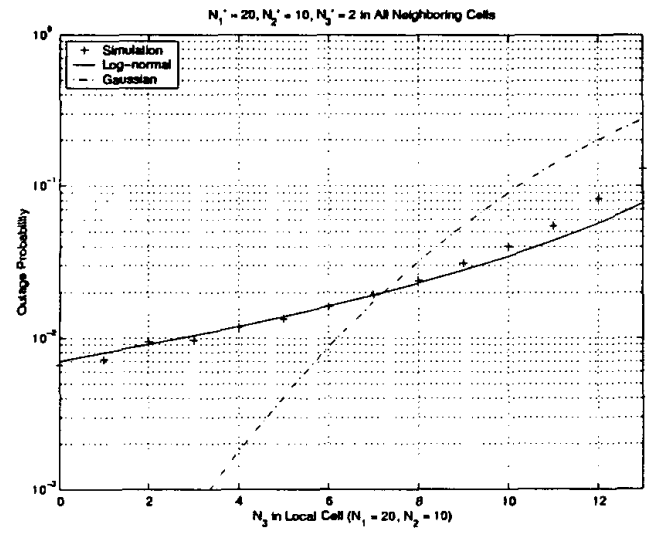

(b) fixed traffic in neighboring cells

Fig. 4. Outage probability with heterogeneous traffic load

[2] A. J. Viterbi, CDMA: Principles of spread spectrum communication, Addison-Wesley Pub., 1995.

[3] G. L. Stüber, Principles of mobile communications, Kluwer Academic Pub., 1996.

[4] A. Baier, U. Fiebig, W. Granzow, W. Koch, P. Teder and J. Thielecke, "Design study for a CDMA-based third-generation mobile radio system," IEEE J. Select. Areas Commun., Vol. 12, No. 4, May 1994, pp. 733-743.

[5] T. Ojanperä and R. Prasad, "An overview of air interface multiple access for IMT-2000/UMTS," IEEE Commun. Mag., Sep. 1998, pp. 82-95.

[6] F. Adachi, M. Sawahashi and H. Suda, "Wideband DS.CDMA for nextgeneration mobile communications systems," IEEE Commun. Mag., Sep. 1998, pp. 56-69.

[7] D. N. Knisely, Q. Li, and N. S. Ramesh, "Cdma2000: A third-generation radio transmission technology," Bell Labs Tech. J., Jul.-Sep. 1998, pp. 63. 78.

[8] A. Sampath and J. M. Holtzman, "Access control of data in integrated voice/data CDMA systems: Benefits and tradeoffs," IEEE J. Select. Areas Commun., vol. 15, no. 8, Oct. 1997, pp. 1511-1526.

[9] C.-N. Wu, Y.-R. Tsai, and J.-F. Chang, "A quality-based birth-and-death queveing model for evaluating the performance of an integrated voice/data CDMA cellular system," IEEE Trans. Vehicul. Tech., vol. 48, no. 1, Jan. 1999, pp. 83-89.

[10] W.-B. Yang and E. Geraniotis, "Admission policies for integrated voice and data traffic in CDMA packet radio networks," IEEE J. Select. Areas Commun., vol. 12, no. 4, May 1994, pp. 654-664.

[11] T.-K. Liu and J. A. Silvester, "Joint admission/congestion control for wireless CDMA systems supporting integrated services," IEEE J. Select. Areas Commun., vol. 16, no. 6, Aug. 1998, pp. 845-857.

[12] J. S. Evans and D. Everitt, "On the teletraffic capacity of CDMA cellular networks," IEEE Trans. Vehicul. Tech., vol. 48, no. 1, Jan. 1999, pp. 153 . 165.

[13] J. S. Evans and D. Everitt, "Effective bandwidth-based admission control for multiservice CDMA cellular networks," IEEE Trans. Vehicul. Tech. vol. 48 , no. 1, Jan. 1999 , pp. $36-46$.

[14] D. G. Jeong, I. G. Kim and D. Kim, "Capacity analysis of spectrally overlaid multiband CDMA mobile networks," IEEE Trans. Vehicul. Tech., vol. 47, No. 3, Aug. 1998, pp. 798-807.

[15] S. Kumar and S. Nanda, "High data-rate packet communications for cellular networks using CDMA: Algorithms and performance," IEEE J. Select Areas Commun., vol. 17, no. 3, Mar. 1999, pp. 472-492.

[16] J. Zou and V. K. Bhargava, "Design issues in a CDMA cellular system with heterogeneous traffic types," IEEE Trans. Vehicul. Tech., vol. 47, no 3, Aug. 1998, pp. 871-884.

[17] M. G. Jansen and R. Prasad, "Capacity, throughput, and delay analysis of a cellular DS CDMA system with imperfect power control and imperfect sectorization," IEEE Trans. Vehicul. Tech., vol. 44, no. 1, Feb. 1995, pp. 67-75. 